\title{
Microscopic analysis of shot-noise suppression in nondegenerate diffusive conductors
}

\author{
T. González, J. Mateos, and D. Pardo \\ Departamento de Física Aplicada, Universidad de Salamanca, Plaza de la Merced s/n, E-37008 Salamanca, Spain \\ O. M. Bulashenko \\ Departament de Física Fonamental, Universitat de Barcelona, Avenida Diagonal 647, E-08028 Barcelona, Spain \\ L. Reggiani \\ Istituto Nazionale di Fisica della Materia, Dipartimento di Scienza dei Materiali, Università di Lecce, Via Arnesano, 73100 Lecce, Italy
}

(Received 4 November 1998; revised manuscript received 21 January 1999)

\begin{abstract}
We present a theoretical investigation of shot-noise suppression due to long-range Coulomb interaction in nondegenerate diffusive conductors. Calculations make use of an ensemble Monte Carlo simulator selfconsistently coupled with a one-dimensional (1D) Poisson solver. We analyze the noise in a lightly doped active region surrounded by two contacts acting as thermal reservoirs. By taking the doping of the injecting contacts and the applied voltage as variable parameters, the influence of elastic and inelastic scattering in the active region is investigated. The transition from ballistic to diffusive transport regimes under different contact injecting statistics is analyzed and discussed. Provided significant space-charge effects take place inside the active region, long-range Coulomb interaction is found to play an essential role in suppressing the shot noise at $q U \gg k_{B} T$. In the elastic diffusive regime, momentum space dimensionality is found to modify the suppression factor $\gamma$, which within numerical uncertainty takes values respectively of about $1 / 3,1 / 2$, and 0.7 in the $3 \mathrm{D}$, $2 \mathrm{D}$, and $1 \mathrm{D}$ cases. In the inelastic diffusive regime, shot noise is suppressed to the thermal value.
\end{abstract}

[S0163-1829(99)02128-1]

\section{INTRODUCTION}

Shot noise is caused by the randomness in the flux of carriers crossing the active region of a given device, and is associated with the discreteness of the electric charge. ${ }^{1-3}$ At low frequency (small compared to the inverse of the transit time through the active region, $f \ll 1 / \tau_{T}$, but sufficiently high to avoid $1 / f$ contributions) the power spectral density of shot noise is given by $S_{I}(0)=\gamma 2 q I$, where $I$ is the dc current, $q$ the electron charge, and $\gamma$ the suppression factor. Uncorrelated carriers exhibiting Poissonian statistics are known to be characterized by a full shot-noise power $(\gamma=1)$. However, correlations between carriers can reduce the noise, leading to suppressed shot noise with $\gamma<1$. Several interactions and mechanisms can introduce correlations among carriers, thus giving rise to different levels of suppression, ${ }^{4}$ which can provide valuable information concerning the carrier kinetics inside the devices not available from dc characteristics or lowfrequency conductance. ${ }^{5}$

In ballistic systems, like vacuum tubes, shot noise is known since the seminal work of Schottky, ${ }^{6}$ and well understood in terms of the Poissonian statistics of injected carriers. Within this model, shot noise has been investigated also in several nonuniform devices like Schottky diodes, p-n junctions, tunnel diodes, etc. ${ }^{2}$ In contrast with these ballistic or quasi-ballistic structures, in macroscopic devices, where scattering mechanisms with phonons, impurities and other carriers determine the transport properties, shot noise is not usually detected and noise levels close to the thermal value are typically measured (in the frequency range beyond $1 / f$ and generation-recombination contributions).

With the recent development of mesoscopic conductors, shot noise is receiving renewed attention. In particular, being a signature of correlations among particles, the phenomenon of suppression has emerged as a subject of relevant interest. The suppression has been predicted theoretically as a consequence of Pauli exclusion principle under strongly degenerate conditions in very different situations. In the ballistic regime, shot noise is completely suppressed ${ }^{7,8}$ due to the non-fluctuating occupation number of incoming states. In a point contact, a peak in the noise is predicted in between the conductance plateaus. ${ }^{8}$ In symmetric double-barrier junctions a $1 / 2$ suppression factor has been theoretically explained by different authors. ${ }^{9-12}$ In the case of elastic diffusive conductors, a $1 / 3$ reduction of the noise has been calculated for noninteracting electrons, ${ }^{11,13,14}$ while in the case of strong electron-electron scattering the value of the suppression factor is $\sqrt{3} / 4 .{ }^{15,16}$ Finally, when the devices become macroscopic and inelastic processes are present, like scattering with phonons, the noise is expected to reduce to the thermal value. ${ }^{17,18}$ Remarkably, many of these predictions have been experimentally confirmed, ${ }^{19-30}$ thus opening new and interesting perspectives. Within this scenario, the understanding of the physical mechanisms originating shot noise and its suppression in mesoscopic conductors, and more generally in small-dimensional devices, is a field of growing interest.

Most of the theoretical works carried out so far consider degenerate conductors, where the Pauli exclusion principle plays a major role, and neglect long-range Coulomb interaction among carriers. The influence of this interaction is known to be relevant to the noise reduction since the times of vacuum tubes, ${ }^{31}$ and its possible role in the case of mesoscopic samples ${ }^{32}$ has been repeatedly claimed by Landauer. ${ }^{33-36}$ However, only recently some works, always 
dealing with degenerate conductors, include explicitly longrange Coulomb interaction ${ }^{37}$ and analyze its influence on the high-frequency spectrum of shot noise. ${ }^{38-42}$ A systematic analysis of shot-noise suppression in nondegenerate conductors with the inclusion of Coulomb correlations is still lacking.

Coulomb interaction can affect noise in two main ways. On the one hand, being responsible for total current conservation, it leads to local voltage fluctuations which try to preserve charge neutrality and as such may influence the current noise. ${ }^{13,32}$ On the other hand, being a repulsive force, it tends to space electrons more regularly than a Poissonian statistics, thus reducing the possible noise present in the current flux. ${ }^{36}$ This regulation of the electron motion is particularly evident in the case of ballistic transport, which was investigated under nondegenerate conditions in previous works. ${ }^{43-45}$ However, to our opinion, the role of Coulomb interaction in suppressing shot noise in the presence of scattering is still not well assessed. Here two issues are of main concern: (i) the determination of the noise reduction under elastic diffusive conditions, and (ii) the understanding of the progressive disappearance of shot noise when passing from the mesoscopic to the macroscopic scale of conduction under the influence of inelastic processes.

The aim of this paper is to shed new light on the above issues by investigating microscopically shot noise and its suppression in nondegenerate conductors in the presence of elastic and inelastic scattering and long-range Coulomb interaction. Our approach differs from those typically used to analyze noise in mesoscopic systems. Calculations are based on an ensemble Monte Carlo (MC) simulation selfconsistently coupled with a Poisson solver (PS). Here the scattering mechanisms and the fluctuations of the selfconsistent potential are intrinsically accounted for. In addition, the approach can analyze different voltage-bias conditions, ranging from thermal equilibrium to high electric fields necessary for shot noise to manifest itself, without the difficulties that other methods meet. ${ }^{37}$ With this approach we investigate shot-noise suppression under the following conditions: (i) crossover between ballistic and diffusive transport regimes and different carrier injecting statistics, (ii) diffusive transport regime under elastic or inelastic scattering, and (iii) 3D, 2D, and 1D momentum space. A MC simulation of shotnoise suppression in a $1 \mathrm{D}$ mesoscopic conductor was performed in Ref. 46. There, the effect of Pauli exclusion principle in the presence of elastic and inelastic scattering in a completely degenerate sample was analyzed, but Coulomb interaction was neglected.

Despite the fact that the physical system we analyze is nondegenerate, some of the results we get coincide with those obtained in degenerate systems by using other approaches. In particular, it is specially surprising that we find the same universal $1 / 3$ value for the shot-noise suppression factor under elastic diffusive regime. ${ }^{47}$ This $1 / 3$ value has been obtained by very different theoretical approaches, going from quantum-phase-coherent ${ }^{13}$ to semiclassical-diffusive degenerate models. ${ }^{11,14,46}$ The universality of this factor has been demonstrated both in quantum ${ }^{48,49}$ and semiclassical contexts. ${ }^{50,51}$ In all these cases degenerate conditions are assumed, and the noise reduction comes from the regulation of electron motion by the exclusion principle. However, it is not

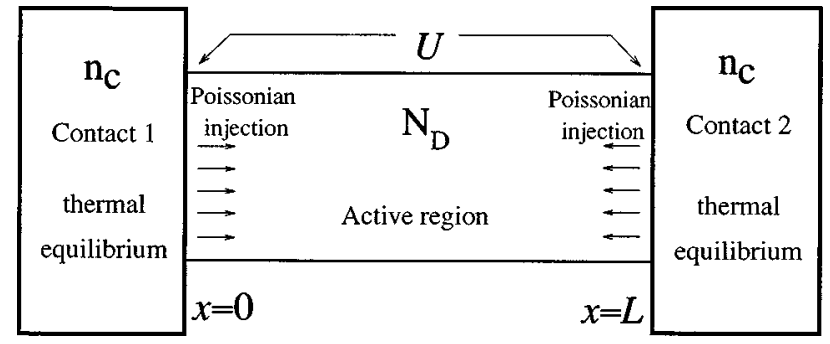

FIG. 1. Schematic drawing of the structure under investigation.

clear whether the different approaches are equivalent, and the reappearance of the $1 / 3$ factor could be just a numerical coincidence, as critically asserted by Landauer. ${ }^{36}$ Here the $1 / 3$ suppression factor appears in another unrelated context where neither phase coherence nor Fermi statistics are present. In our case the origin of the effect is completely classical, and the correlation between electrons comes just from their Coulomb repulsion. ${ }^{47}$ Moreover, we show that the $1 / 3$ value only appears when a $3 \mathrm{D}$ momentum space is considered. In the 2D or 1D cases different factors are obtained, though the physical mechanism of suppression remains the same. To this purpose, an analytical theory which explains this dependence of the suppression factor on the dimension of momentum space has been recently developed by Beenakker. ${ }^{52}$ Finally, we also illustrate the essential role played by Coulomb interaction for the suppression of shot noise by inelastic scattering, as already stressed by Büttiker. $^{32}$

The outline of the paper is as follows. In Sec. II we discuss the physical model used for the structures under analysis. Section III presents the results of shot-noise suppression with reference to (i) the crossover between ballistic and diffusive transport regimes, (ii) the elastic and inelastic diffusive regime, and (iii) the role of momentum-space dimensionality. In Sec. IV the main conclusions and future trends are briefly surveyed.

\section{PHYSICAL MODEL}

For the present analysis we consider the simple structure shown in Fig. 1. It consists of a lightly doped active region of a semiconductor sample sandwiched between two heavily doped contacts (of the same semiconductor) which act as thermal reservoirs and inject carriers into the active region. The sample is assumed to have a transversal size sufficiently thick to allow a 1D electrostatic treatment in the $x$ direction and to neglect the effects of boundaries in $y$ and $z$ directions. The doping of the contacts $n_{c}$ is taken to be much higher than that of the active region $N_{D}$. The carrier density at the contacts corresponds to their doping concentration; all impurities are assumed to be ionized at the temperature $T$ $=300 \mathrm{~K}$ considered here. The contacts are assumed to have no voltage drop inside and to remain always at thermal equilibrium. Accordingly, when a voltage $U$ is applied to the structure, all the potential drop takes place inside the active region, between the positions $x=0$ and $x=L$. The transport analysis is carried out by simulating the carrier dynamics only in the active region of the structure by using an ensemble MC technique self-consistently coupled with a PS. ${ }^{53}$ The influence of the contacts is included by means of a sto- 
chastic injection rate taking place at positions $x=0$ and $x$ $=L$. The simulation is $1 \mathrm{D}$ in real space, the Poisson equation being solved in the direction $x$ of the applied voltage. Typically, a 3D momentum space is considered. However, to analyze the influence of dimensionality on the noise suppression, a 2D and 1D momentum space is also considered in some specific cases. Static (i.e., frozen electric field profile) and dynamic PS schemes are used to analyze the importance of Coulomb correlations. The details of the MC modeling can be found in Refs. 43 and 45 .

For the calculations we have used the following set of parameters: carrier effective mass $m=0.25 m_{0}$, dielectric constant $\varepsilon=11.7 \varepsilon_{0}$ ( $\varepsilon_{0}$ being the vacuum permittivity), $L$ $=2000 \AA, n_{c}$ ranging between $10^{13} \mathrm{~cm}^{-3}$ and $10^{18} \mathrm{~cm}^{-3}$, and $N_{D}=10^{11} \mathrm{~cm}^{-3}$. The injection rate at the contacts, $\Gamma$, is proportional to $n_{c}$ and determines the level of space charge inside the active region, which is characterized by the dimensionless parameter $\lambda$, defined as ${ }^{43,45}$

$$
\lambda=\frac{L}{L_{D c}},
$$

where $L_{D c}=\sqrt{\varepsilon k_{B} T / q^{2} n_{c}}$ is the Debye length corresponding to the carrier concentration at the contacts. In present calculations $\lambda$ takes the minimum value of $0.15 \quad\left(n_{c}\right.$ $=10^{13} \mathrm{~cm}^{-3}$ ), for which the effects of Coulomb repulsion between electrons are practically negligible, and the maximum values of $30.9\left(n_{c}=4 \times 10^{17} \mathrm{~cm}^{-3}\right)$ and $48.8\left(n_{c}\right.$ $\left.=10^{18} \mathrm{~cm}^{-3}\right)$, for which quite significant electrostatic screening takes place.

As already indicated, the structures we consider contain space charge in the active region, and thus total charge neutrality is ensured by the external circuit, here represented by the contacts. In our model, due to the very high value of $n_{c}$ as compared to $N_{D}$, all the band bending is assumed to take place exclusively in the active region under any applied bias. ${ }^{54}$ For the same reason, any possible influence of the applied voltage (especially for high values) on the contacts and, consequently, on the injection rate $\Gamma$, is neglected. Accordingly, the boundary condition for carrier injection is described through a constant $\Gamma$, whose value is determined by $n_{c}$. With this ideal model for the contacts, the structure acts similarly to a vacuum tube (with $\Gamma$ regulated by $\left.n_{c}\right)^{55}$ with the relevant differences that: (i) scattering mechanisms can take place, and (ii) two injecting contacts, and therefore two opposing currents which flow in the presence of a medium, are considered.

Scattering mechanisms are introduced in the simulation in a simple way by making use of an energy independent relaxation time $\tau$. We consider separately elastic and inelastic (completely thermalizing) interactions, both taken to be isotropic. ${ }^{47}$ While $L$ remains constant, the value of $\tau$ is appropriately varied from 10 ps to 1 fs thus covering both the ballistic and diffusive transport regimes. The transition between these regimes is characterized by the ratio between the carrier mean free path $\ell$ and the sample length $L$. Typical values of the time step and number of meshes in real space used for the PS are 2 fs and 100, respectively, except for the cases when $\tau<5$ fs for which the time step is taken of $0.2 \mathrm{fs}$. As test of numerical reliability we have checked that by reducing the time step or by increasing the number of meshes the results remain the same. The average number of simulated particles in the active region ranges between 50 and 2000 depending on contact doping, transport regime, and applied voltage. From the sequence of instantaneous values of the current $I(t)$ obtained from the MC simulation ${ }^{43}$ we firstly calculate the autocorrelation function of current fluctuations $C_{I}(t)$, and then, by Fourier transformation, the spectral density $S_{I}(f)$. The suppression factor is then evaluated as $\gamma=S_{I}(0) / 2 q I$. To distinguish between the results obtained from the static and dynamic PS, we denote the corresponding current spectral densities as $S_{I}^{S}$ and $S_{I}^{d}$, respectively.

Unless otherwise indicated, calculations make use of a Poissonian-Maxwellian contact model for carrier injection, described in detail in Ref. 45, which appears to be physically plausible under nondegenerate conditions. However, to analyze the influence of the contact injecting statistics on the noise behavior, alternative models are also used. In particular, for the injected carriers we consider: (i) fixed velocity instead of Maxwellian distribution and, (ii) uniform-in-time instead of Poissonian injection. In case (i) we consider the same injection rate $\Gamma$ as in the basic model, but all carriers are injected with identical $x$-velocity $\mathrm{v}_{x}=\sqrt{\pi k_{B} T / 2 m}$, which corresponds to the average velocity of the injected electrons when they follow a Maxwellian distribution. In case (ii) carriers are injected into the active region equally spaced in time at intervals of $1 / \Gamma$.

\section{RESULTS}

The main results of the present work are organized as follows. Subsections A and B pertain to a 3D momentum space while subsection $\mathrm{C}$ is devoted to $2 \mathrm{D}$ and $1 \mathrm{D}$ momentum spaces. Most of the reported results refer to high values of the space-charge parameter $\lambda$ (typically $\lambda=30.9$ ), which implies the presence of significant effects related to longrange Coulomb interaction in the active region of the structures. ${ }^{56}$ The essential influence of $\lambda$ on the suppression factor $\gamma$ is analyzed at the end of subsection $\mathrm{B}$. We recall that elastic and inelastic scattering are considered separately in the simulations. In no case both types of scattering are taken into account simultaneously.

\section{A. Transition ballistic-diffusive regimes}

The behavior of noise in the crossover from ballistic to diffusive transport regimes is analyzed under far-fromequilibrium conditions $\left(U \gg k_{B} T / q\right)$, since these are necessary for the manifestation of shot noise. The dependence of noise on the applied voltage for the perfect ballistic regime was already reported in Ref. 45 and for the perfect diffusive regime it will be analyzed in the next subsection.

Figure 2 reports the variance of carrier-number fluctuations normalized to the average carrier number $\left\langle\Delta N^{2}\right\rangle /\langle N\rangle$ as a function of $\ell / L$. The values taken by the variance are found to be practically independent of the transport regime. The results of the elastic and inelastic cases are very similar; by contrast two different values are obtained depending on the scheme of the PS used in the calculations. When the static PS is considered, within numerical uncertainty ${ }^{57}$ it is found that $\left\langle\Delta N^{2}\right\rangle=\langle N\rangle$ in all the range of $\ell / L$ reported. 


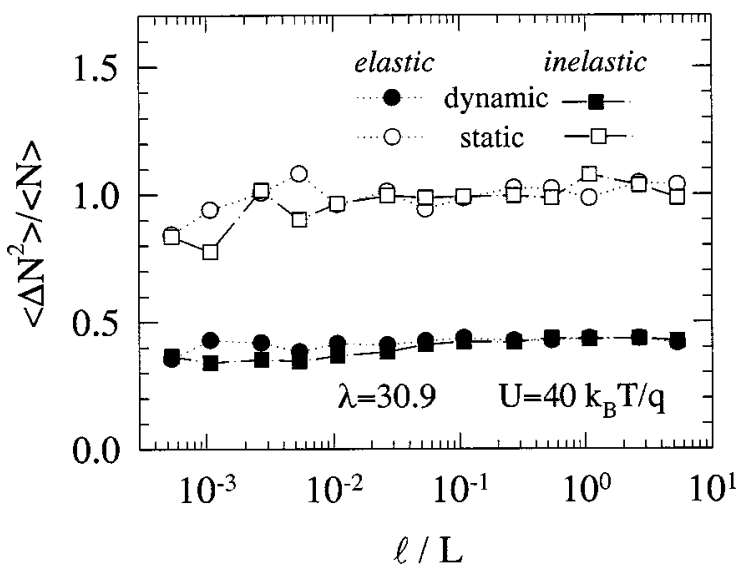

FIG. 2. Variance of carrier number inside the active region normalized to the average carrier number vs the ballistic parameter $\ell / L$ for an applied voltage of $U=40 k_{B} T / q$. Calculations are performed by using static and dynamic PS schemes, and considering elastic and inelastic scattering mechanisms.

Therefore, in the absence of dynamic Coulomb correlations, the carrier number follows a Poissonian statistics. In the perfect ballistic regime this is a consequence of the injecting statistics at the contacts. In the diffusive regime this is due to the effect of the randomness introduced by scattering, independently of its elastic or inelastic property. Because of the Poissonian statistics observed for the carrier-number fluctuations, no shot-noise suppression is expected within the static PS scheme. On the contrary, when the dynamic PS scheme is used, we clearly observe a sub-Poissonian behavior with $\left\langle\Delta N^{2}\right\rangle$ being about a factor of 0.4 lower than $\langle N\rangle$; thus shot-noise suppression is expected.

Figure 3 reports $S_{I}(0)$ normalized to $2 q I_{S}$, with $I_{S}=q \Gamma$ the saturation current of the contact, ${ }^{45}$ under the same conditions of Fig. 2. Here the evolution of the current in terms of $2 q I$ is also shown. This evolution exhibits two limiting behaviors, namely: saturation at $\ell / L \geqslant 10^{-1}$, typical of a ballistic or quasi-ballistic regime; and linear decrease at $\ell / L$ $\lesssim 10^{-2}$ as $\ell / L \rightarrow 0$, typical of a diffusive regime. In both the

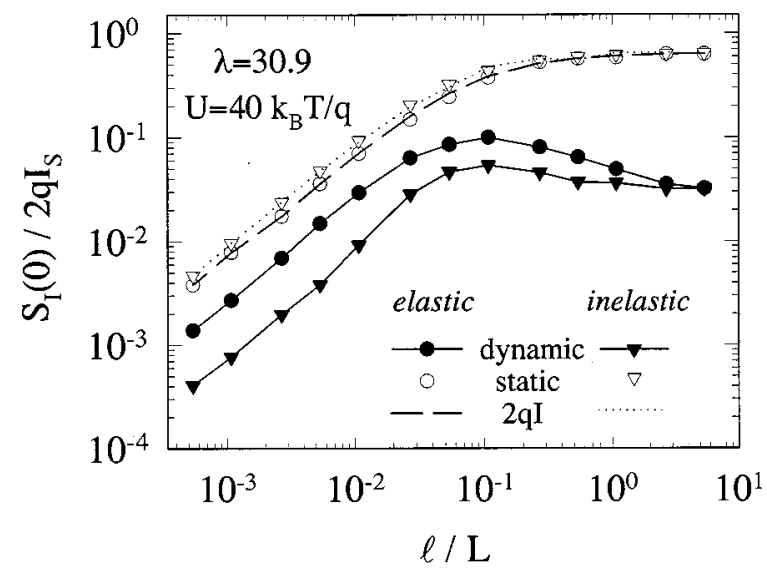

FIG. 3. Low-frequency spectral density of current fluctuations normalized to $2 q I_{S}$ vs the ballistic parameter $\ell / L$ for an applied voltage of $U=40 k_{B} T / q$. Calculations are performed by using static and dynamic PS schemes, and considering elastic and inelastic scattering mechanisms. The dependence of the current through $2 q I$ is also plotted for comparison.

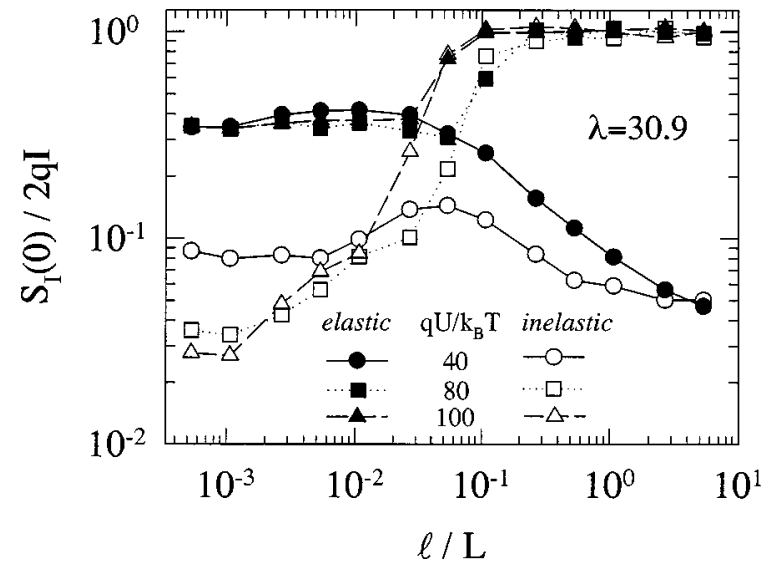

FIG. 4. Shot-noise suppression factor vs ballistic parameter $\ell / L$ for the cases of elastic and inelastic scattering at different applied voltages. Calculations are performed by using the dynamic PS scheme.

elastic and inelastic cases, $S_{I}(0)$ calculated with the static PS coincides exactly with $2 q I,{ }^{58}$ thus leading to the conclusion that even in the presence of intensive scattering processes (no matter elastic or inelastic), the stream of uncorrelated carriers exhibits full shot noise behavior like in the perfect ballistic regime. On the contrary, with the dynamic PS $S_{I}(0)$ is systematically lower than $2 q I$, thus evidencing a suppression effect. Here, as expected, elastic and inelastic curves recover the same value in the ballistic limit, the suppression being caused by the fluctuations of the potential barrier near the cathode (induced by the space charge) which controls the current in this regime. ${ }^{43-45}$ By approaching the perfect diffusive conditions, the suppression remains active, more pronounced in the inelastic case, and is related to the joint action of the Coulomb repulsion and the presence of scattering.

We now consider only the dynamic PS scheme. Figure 4 reports $\gamma$ as a function of $\ell / L$ for several values of the applied voltage. In the perfect ballistic regime the two distinct values of $\gamma$ so found refer to the presence or absence of the potential barrier due to space charge. ${ }^{45}$ For $U=40 k_{B} T / q$ the barrier is still present and the suppression is important. For $U=80$ and $100 k_{B} T / q$ the barrier has already disappeared; accordingly the current saturates and the suppression factor takes on the full shot-noise value. When the diffusive regime is achieved, in the elastic case $\gamma$ attains a constant value at further decreasing of $\ell / L$, and takes the same value of about $1 / 3$ independently of the applied voltage. On the contrary, in the inelastic case the higher the applied voltage the lower the value which $\gamma$ is found to take. Remarkably, when the ballistic regime is abandoned the value of $\ell / L$ at which $\gamma$ starts decreasing is the same in the elastic and inelastic cases for a given applied voltage $(\ell / L \approx 0.3$ and 0.1 , for 80 and 100 $k_{B} T / q$, respectively). However, when the diffusive regime is approached, for $\gamma$ to become constant a lower value of $\ell / L$ must be reached in the inelastic case with respect to the elastic one. This behavior can be explained in terms of the different elastic and inelastic scattering intensity required by the electron system to achieve a significant energy equipartition into the three directions of momentum space.

To better illustrate the above features, Fig. 5 shows the spatial profiles of the total average energy and its three contributions along the $x, y$ and $z$ directions of momentum space 

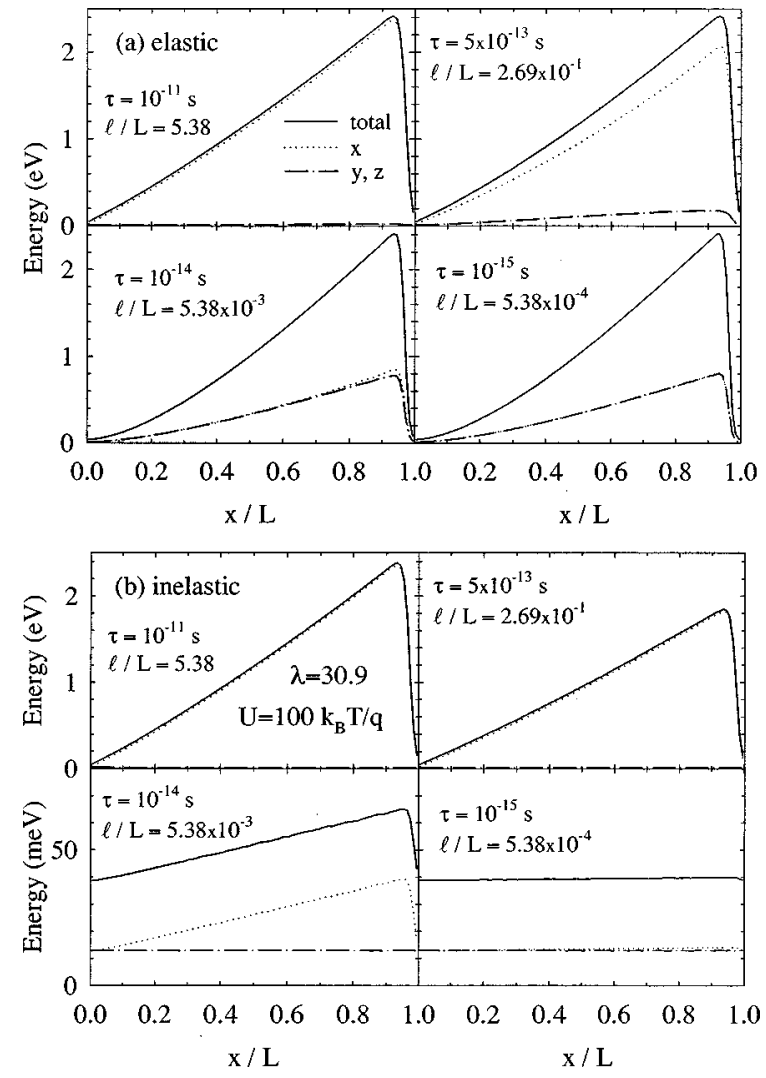

FIG. 5. Spatial profiles along the sample of the total average energy and its three contributions in the $x, y$, and $z$ directions of momentum space for several values of the scattering time $\tau$ (and of the associated $\ell / L)$. The applied voltage is $U=100 k_{B} T / q$. (a) refers to elastic scattering and (b) to inelastic scattering.

for several values of $\tau$ in the elastic and inelastic cases at $U=100 k_{B} T / q$. For the longest $\tau$, the total energy practically coincides with the energy in the $x$ direction independently of elastic and inelastic cases, since the transport is nearly ballistic. In the case of elastic scattering [Fig. 5(a)], as $\tau$ is shortened the energy in the $x$ direction decreases while that in the other two directions increases, in such a way that the total energy remains constant, as follows from elastic conditions. By further shortening $\tau$, the energy in the three directions becomes finally the same and equipartition condition is achieved. In the inelastic case [Fig. 5(b)], equipartition of energy is also reached, but only at the shortest $\tau$. The fact that energy equipartition is more easily reached in the elastic than in the inelastic case is understood as follows. The occurrence of an elastic scattering distributes to the $y$ and $z$ directions part of the energy that the carrier initially had in the $x$ direction, so that the energy tends to equalize in the three momentum components. However, for the case of an inelastic scattering only the energy in the $x$ direction changes, since the energy in the $y$ and $z$ directions always corresponds to that of the thermal equilibrium distribution $\left(\frac{1}{2} k_{B} T\right)$. This means that for a given applied voltage, a higher number of scattering events (i.e., a lower value of $\ell(L)$ is necessary to achieve energy equipartition in the inelastic case with respect to the elastic one. Thus, in Fig. 5(a) it can be observed that for $\ell / L=5.38 \times 10^{-3}$ and an applied voltage of $100 k_{B} T / q$, in the elastic case an important isotropic redistribution of energy has already been achieved, so

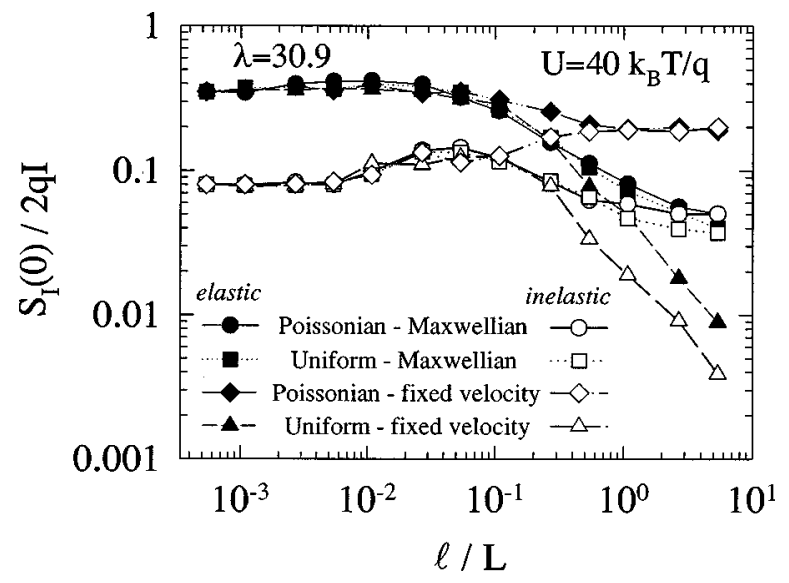

FIG. 6. Shot-noise suppression factor vs the ballistic parameter $\ell / L$ for an applied bias of $U=40 k_{B} T / q$ calculated with different contact models. Calculations refer to the dynamic PS scheme considering elastic and inelastic scattering.

that $\gamma$ already takes the value $1 / 3$ (Fig. 4). However, in the inelastic case [Fig. 5(b)], for the same value of $\ell / L$ the energy in the $x$ direction is still significantly higher than that in the other two directions of momentum space and, as a result, $\gamma$ has not still reached its constant value (which corresponds to thermal noise, as shown later) and continues decreasing. These results confirm that thermal conditions, and therefore thermal equilibrium noise, are not reached until the inelastic scattering time is so short that the energy gained by electrons in a mean free path is much lower than the thermal energy, as already indicated by Landauer. ${ }^{35}$

To analyze the role played by the modeling of the contact injection on the suppression of noise, Fig. 6 reports $\gamma$ as a function of $\ell / L$ calculated using four different models. They combine Poissonian/uniform injecting statistics and Maxwellian/fixed-velocity distribution of the injected carriers. The Poissonian-Maxwellian injection is the basic one used in calculations. In the perfect ballistic regime, when carrier transport is deterministic, $\gamma$ crucially depends on the injection model. Thus, in the case of the uniform fixedvelocity model, when the injection introduces no extra noise in the current flux, $\gamma$ is found to decrease linearly with the increase of $\ell / L$. The noise does not vanish completely since, unless $\ell / L \rightarrow \infty$, there is always some probability of undergoing a scattering event. In this limit, when the noise is produced just by a few scattering events, it is clearly observed that elastic interactions lead to more important current fluctuations than inelastic mechanisms. By approaching the perfect diffusive regime the suppression factor is found to be independent of the model used. We conclude that the noise in the diffusive regime (and particularly the 1/3 suppression value obtained in the elastic diffusive case) is independent of the carrier injecting statistics, and it is only determined by the joint action of scattering events and Coulomb correlations.

\section{B. Diffusive regime}

In this section we consider scattering times short enough to ensure a diffusive transport regime $\left(\ell / L \leq 3 \times 10^{-3}\right)$. In this regime the noise behavior is closely related to the breadth of the velocity distribution, ${ }^{15,35}$ as shown for the 


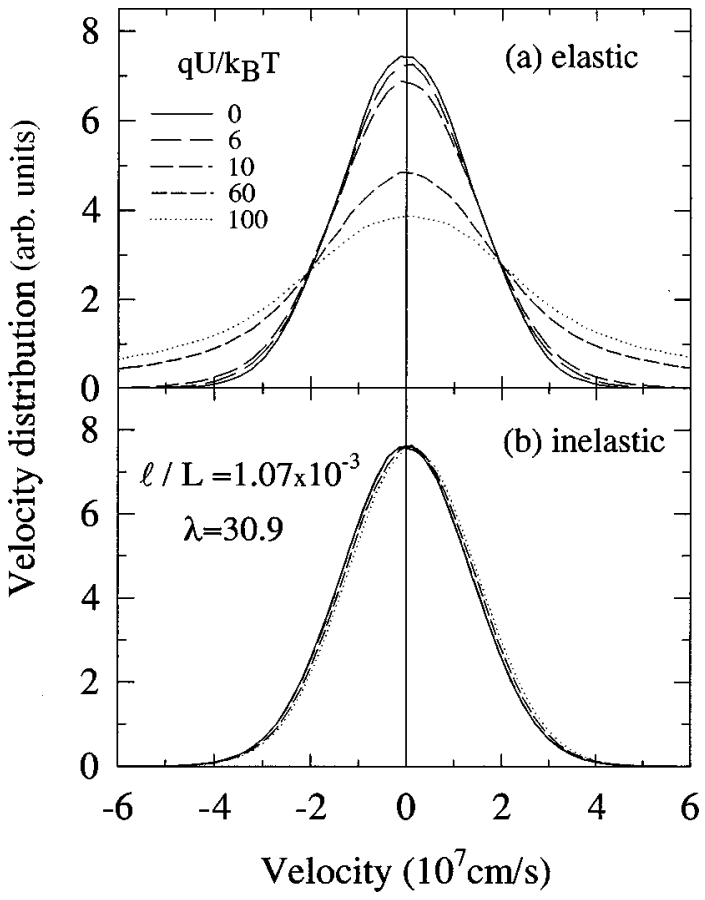

FIG. 7. Velocity distribution function of carriers inside the active region of the structure for $\ell / L=1.07 \times 10^{-3}$ at several applied voltages. (a) refers to elastic scattering and (b) to inelastic scattering.

$x$-velocity component in Fig. 7. In the case of elastic scattering, the distribution broadens at increasing applied voltages since there is no energy dissipation. In the inelastic case, the energy is maximally dissipated by recovering the thermal value after each scattering event; thus a thermal equilibrium Maxwell-Boltzmann distribution is obtained independently of the applied voltage. In both cases the distributions are very slightly displaced to positive velocities, as implied by the presence of a net current flowing through the structure. We remark that the distributions shown in Fig. 7 refer to all the carriers present inside the sample. In the elastic case, the local distributions at given positions are found to exhibit a nearly isotropic but not a Maxwellian shape, however their spatial integration over the whole sample length gives the Maxwellian profile reported in the figure.

Figure 8 shows $S_{I}(0)$ normalized to $2 q I_{S}$ as a function of the applied voltage. Here, the $I-U$ characteristic is also shown in terms of $2 q I$. In the wide range of voltages reported in the figure, ${ }^{59}$ the $I-U$ curve exhibits a super-linear behavior which is related to the importance of space-charge effects and the increase of the carrier number inside the active region with the applied voltage for $q U \gtrsim k_{B} T$. For the highest applied voltages it is found $I \propto U^{r}$, where the exponent $r$ is a function of $\lambda$. In particular, $r=1.7$ for $\lambda=30.9$ (the case shown in the figure) and $r=1.8$ for $\lambda=48.8$. In what concerns $S_{I}(0)$, near thermal equilibrium conditions $\left(q U<k_{B} T\right)$, elastic and inelastic cases exhibit the same value which satisfies Nyquist relation. In the inelastic case, at increasing voltages $S_{I}(0)$ remains practically constant, in accordance with the velocity distributions of Fig. 7(b). The slight increase shown at the highest voltages is strictly related to the increase of the carrier number. On the contrary, in the elastic case at high voltages $\left(q U / k_{B} T \gtrsim 10\right)$, when the

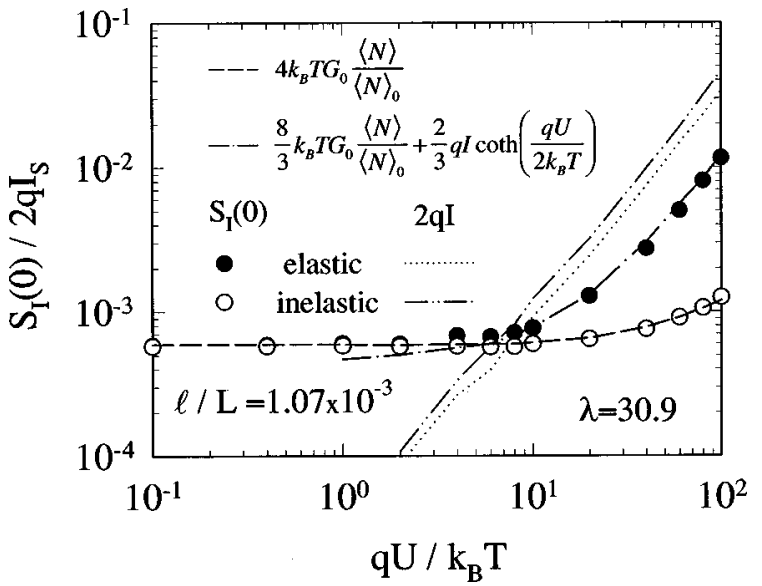

FIG. 8. Low-frequency spectral density of current fluctuations normalized to $2 q I_{S}$ vs applied voltage for $\ell / L=1.07 \times 10^{-3}$. Calculations refer to the dynamic PS scheme considering elastic and inelastic scattering. The current in terms of $2 q I$ is also plotted for comparison. The fittings of Eqs. (2) and (4) are shown by dashed and dash-dotted lines, respectively.

velocity distribution starts broadening significantly from its thermal equilibrium shape [Fig. 7(a)], $S_{I}(0)$ increases systematically with $U$, its ratio to the current remaining constant and providing a value of $1 / 3$ for the suppression factor $\gamma^{47}$

The MC results in the inelastic case $S_{I}^{\text {inel }}(0)$ are closely fitted by the expression

$$
S_{I}^{\text {inel }}(0)=4 K_{B} T G_{0} \frac{\langle N\rangle}{\langle N\rangle_{0}},
$$

where

$$
G_{0}=\frac{q^{2}\langle N\rangle_{0} \tau}{m L^{2}}
$$

is the conductance and $\langle N\rangle_{0}$ the average number of electrons inside the sample, both in the limit of vanishing bias. ${ }^{60} \mathrm{We}$ conclude that inelastic scattering strongly suppresses shot noise and makes the noise become macroscopic $(\gamma \ll 1) .{ }^{47}$ We remark that present findings prove also that the condition of inelastic scattering alone does not suffice to suppress shot noise; the presence of the fluctuating self-consistent electric field remaining a necessary condition. Indeed, as a counterproof we refer to the calculations performed with the static PS scheme, where no suppression has been detected (see Fig. $3)$. Therefore, as argued by Büttiker ${ }^{32}$ it is the combination of both Coulomb interaction and inelastic scattering which leads to the suppression of shot noise. In the elastic case, the values of $S_{I}^{\mathrm{el}}(0)$ are nicely reproduced by the following expression:

$$
S_{I}^{\mathrm{el}}(0)=\frac{8}{3} K_{B} T G_{0} \frac{\langle N\rangle}{\langle N\rangle_{0}}+\frac{2}{3} q I \operatorname{coth}\left(\frac{q U}{2 k_{B} T}\right),
$$

which is quite similar to that obtained by Nagaev ${ }^{14}$ in a degenerate context, and describes the crossover from thermalNyquist noise for $q U \ll k_{B} T$ to $1 / 3$-suppressed shot noise for $q U \gg k_{B} T$. In contrast with other approaches, ${ }^{11,13,14,46}$ our results show that neither phase-coherence ${ }^{50,51}$ nor degenerate statistics are required for the occurrence of suppressed shot 


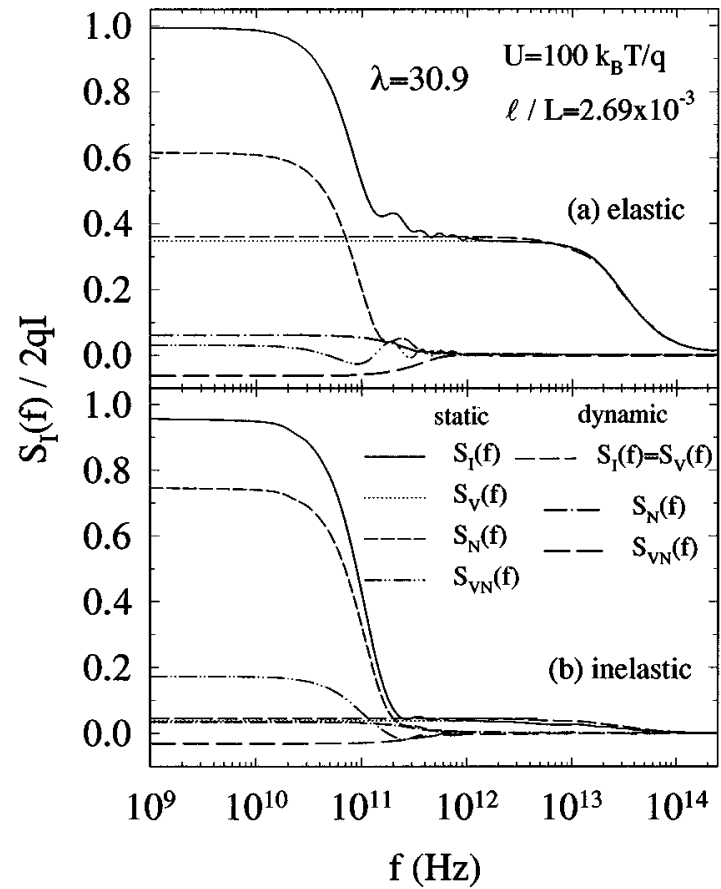

FIG. 9. Spectrum of the shot-noise suppression factor under diffusive regime $\left(\ell / L=2.69 \times 10^{-3}\right)$ calculated within static and dynamic PS schemes for (a) elastic and (b) inelastic cases and an applied voltage of $U=100 k_{B} T / q$. Different contributions to the total spectrum are reported in the figure.

noise in diffusive conductors, and purely classical physical processes can lead to the same $1 / 3$ factor. ${ }^{5}$

To illustrate the physical origin of the $1 / 3$ value, Fig. 9(a) reports a typical spectrum of the suppression factor under elastic diffusive conditions for static and dynamic PS schemes. Here the current spectrum is decomposed into velocity, number, and cross-correlation contributions, $S_{I}(f)$ $=S_{V}(f)+S_{N}(f)+S_{V N}(f){ }^{43,53,61}$ In the static PS scheme the spectrum clearly shows that the three terms contribute to $S_{I}(f)$, and two different time scales can be identified. The longest one is associated with the transit time of carriers through the active region $\tau_{T} \approx 5 \mathrm{ps}$, and is evidenced in the terms $S_{N}^{s}(f)$ and $S_{V N}^{s}(f)$. The shortest one is related to the relaxation time of elastic scattering $\tau=5 \mathrm{fs}$, and is manifested in $S_{V}^{s}(f)$. Remarkably, the velocity contribution yields $1 / 3$ of the full shot-noise value, while the other two terms provide the remaining $2 / 3$. Thus, in the static PS scheme full shot noise is recovered as sum of all the three contributions. On the contrary, in the dynamic PS scheme $S_{N}^{d}(f)$ and $S_{V N}^{d}(f)$ are found to compensate each other and, as a result, $S_{I}^{d}(f)$ coincides with $S_{V}^{d}(f)$ in all the frequency range. Moreover, $S_{N}^{d}(f)$ takes values much smaller than $S_{N}^{s}(f)$. The characteristic time scale of $S_{N}^{d}(f)$ and $S_{V N}^{d}(f)$ differs from that found within the static PS scheme, which was related to the transit time $\tau_{T}$. Now in the dynamic case it is the dielectric relaxation time corresponding to the carrier concentration at the contacts $\tau_{d}=0.46 \mathrm{ps}$ which determines the cutoff of the contributions belonging to number fluctuations. In the frequency range between the transit and collision frequency values it is interesting to notice that both static and dynamic PS schemes yield $\gamma=1 / 3$, thus relating the suppression factor to velocity fluctuations only. How-

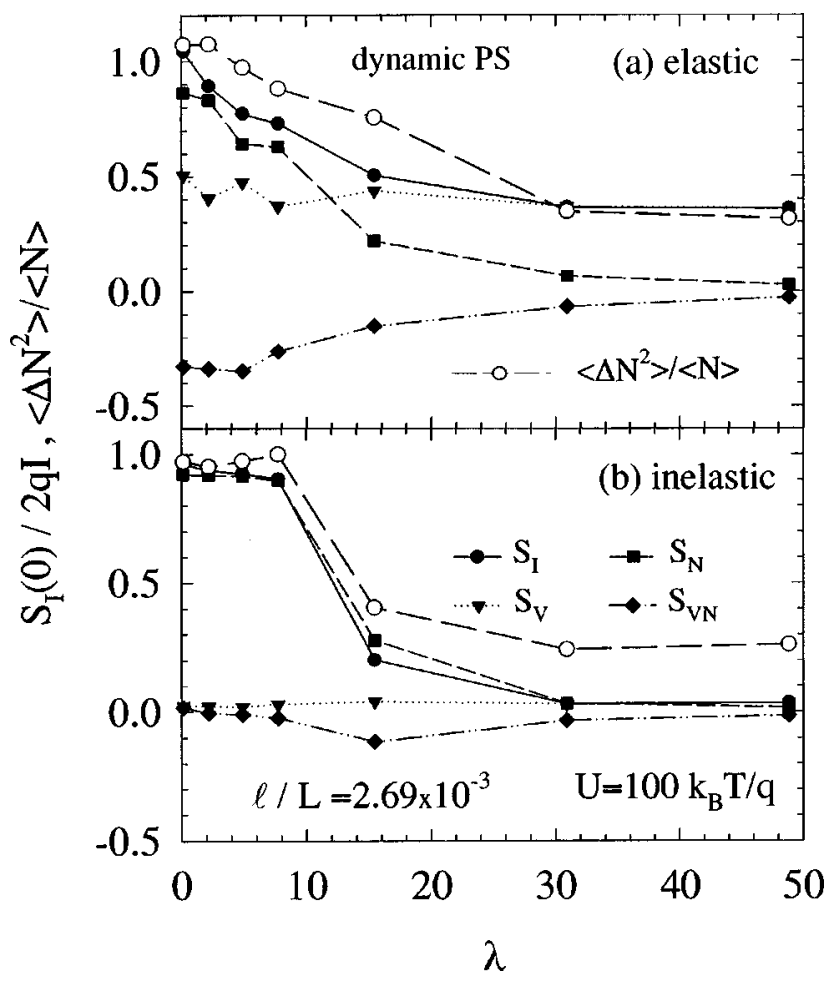

FIG. 10. Shot-noise suppression factor and variance of the carrier number inside the active region as a function of the characteristic parameter of space charge $\lambda$ under diffusive regime $(\ell / L$ $=2.69 \times 10^{-3}$ ) calculated within dynamic PS scheme for (a) elastic and (b) inelastic scattering. Velocity, number, and velocity-number contributions to the suppression factor are also shown in the figure.

ever, at low frequencies only the dynamic scheme takes this value by virtue of Coulomb correlations, which are responsible for the reduction of $S_{N}^{d}(f)$ and the mutual compensation of $S_{N}^{d}(f)$ and $S_{V N}^{d}(f)$ contributions. It is remarkable that $S_{V}^{S}(f)=S_{V}^{d}(f)$ in all the frequency range, which implies that velocity fluctuations are not affected by long-range Coulomb interaction, but just by scattering mechanisms. Coulomb repulsion affects only the contributions where carrier-number fluctuations are involved. Figure 9(b) reports the spectrum for inelastic scattering. Here, the same features of the elastic case are observed, with the important difference that $S_{V}^{d}(f)$ is much lower than when there is no energy dissipation.

So far we have analyzed structures where Coulomb repulsion plays an important role (i.e. $\lambda \gg 1$ ). To check to which extent this interaction is determinant for noise suppression, Fig. 10 reports $\gamma$ and the three contributions into which it has been decomposed as a function of $\lambda$. Here, we present both the elastic and inelastic cases calculated under far-fromequilibrium conditions within the dynamic PS scheme. The variance of the number of carriers inside the sample is also reported to analyze the evolution of carrier statistics as a function of $\lambda$. For the lowest values of $\lambda$, when space-charge effects and in turn Coulomb interaction are negligible, full shot noise is observed. As $\lambda$ increases, $\gamma$ starts decreasing from unity until reaching a constant value for $\lambda \gtrsim 30$. It is remarkable that the contribution of velocity fluctuations to $\gamma$ does not vary significantly with $\lambda$, being much smaller in the inelastic case with respect to the elastic one because of the quasi-thermal conditions imposed by energy dissipation. On 
the contrary, the contributions associated with number and velocity-number fluctuations are strongly affected by the increase of $\lambda$. Indeed, their absolute value decreases systematically and, being opposite in sign, they compensate each other at the highest values of $\lambda$, so that $S_{I}=S_{V}$. We also notice that the transition from full shot noise to suppressed shot noise regimes is sharper in the inelastic case. The variance of the carrier number inside the sample resembles the behavior of $\gamma$, indicating that the increase of $\lambda$ leads to more pronounced sub-Poissonian statistics until reaching the final regime for $\lambda \gg 1$. Therefore, we point out that within our model the $1 / 3$ value exhibits several universal properties, namely, it is independent of (i) the scattering strength (once $\ell \ll L$ ); (ii) the applied voltage (once $q U \gg k_{B} T$ ), (ii) the screening length (once $\lambda \gg 1$ ), and (iv) the carrier injecting statistics.

\section{Dependence on momentum space dimensionality}

The results reported so far refer to a 3D momentum space. In contrast to degenerate diffusive systems where, provided quasi-one-dimensional conditions in real space are attained, noise suppression is independent of the number $d$ of momentum space dimensions, an interesting feature of nondegenerate diffusive systems is that noise suppression can depend on $d$. For the inelastic case considered here no dependence on $d$ has been found, since there is no influence of the velocity components transversal to the electric field direction on transport and noise properties of the structures. On the contrary, in the elastic case the suppression factor is found to depend significantly on $d,{ }^{47}$ since the transversal velocity components constitute a channel for energy redistribution which affects the transport properties of the structure. Therefore, below we focus our analysis on the elastic case. Accordingly, when $d=2$ in the simulation the carrier velocity is randomized in two components after each scattering event, and when $d=1$ the isotropic character of scattering is accomplished by inverting the carrier velocity with an average (back-scattering) probability $P_{b}=0.5$.

Figure 11(a) reports $\gamma$ as a function of $\ell / L$ for the 1D, $2 \mathrm{D}$, and $3 \mathrm{D}$ cases at high voltages $\left(U=40 k_{B} T / q\right)$ calculated within the dynamic PS scheme. We notice that, when calculated within the static PS scheme, the results in 1D and 2D cases do not exhibit any shot-noise suppression, like in the $3 \mathrm{D}$ case. For the highest values of $\ell / L$, in all three cases $\gamma$ approaches the asymptotic value corresponding to the ballistic limit $(\gamma=0.045),{ }^{43}$ where the behavior is independent of $d$. At a given value of $\ell / L$, a higher deviation from the asymptotic ballistic value is observed for lower $d$. This is due to the fact that, in average, elastic interactions introduce higher fluctuations of the carrier $x$-velocity the lower is the number of available momentum states after the scattering mechanism (in particular, just 2 in the 1D case). For this same reason, the increasing presence of scattering as $\ell / L$ is reduced leads to higher values of the suppression factor the lower is the dimensionality. Remarkably, within numerical uncertainty the limit value reached by $\gamma$ in the perfect diffusive regime is found to be, respectively, of $1 / 3,1 / 2$, and 0.7 for $3 \mathrm{D}, 2 \mathrm{D}$, and $1 \mathrm{D} .{ }^{62}$ Figure $11(\mathrm{~b})$, by reporting $\gamma$ in the diffusive regime as a function of the applied voltage, provides evidence that these limit values are independent of the bias once $q U \gg k_{B} T$. The origin of the suppression is the
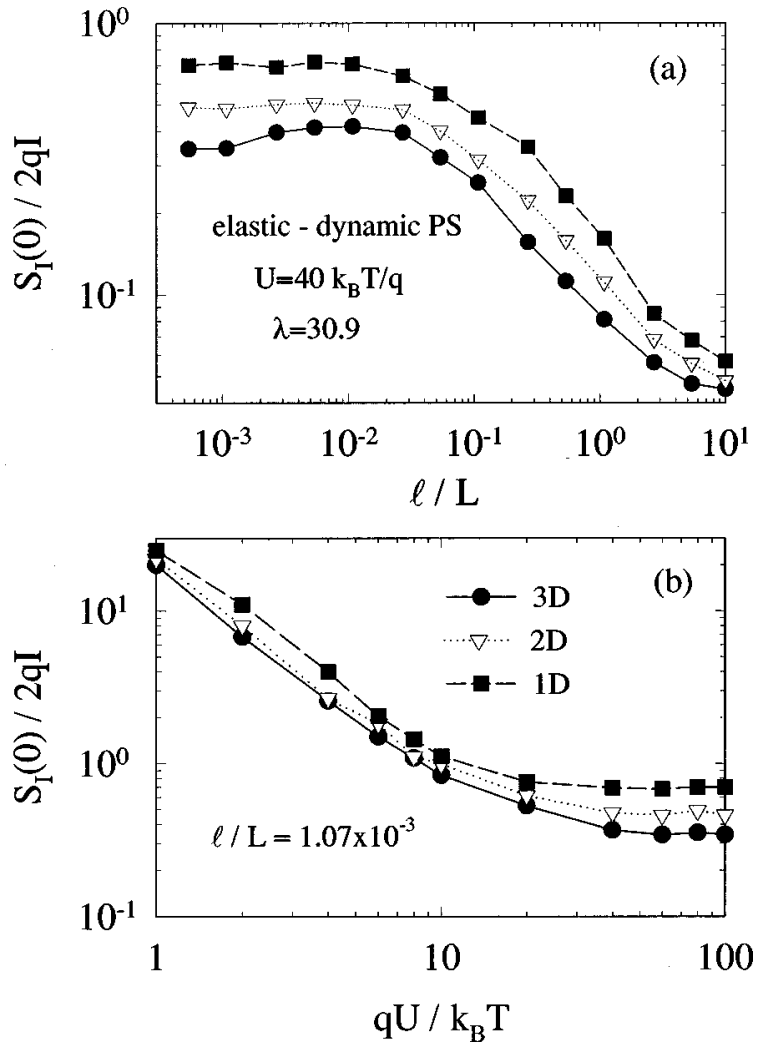

FIG. 11. Shot-noise suppression factor for the cases of 1, 2, and 3 dimensions of momentum space calculated within the dynamic PS scheme for elastic scattering as a function of (a) ballistic parameter $\ell / L$ with an applied voltage of $U=40 k_{B} T / q$ and (b) applied bias $U$ under diffusive regime $\left(\ell / L=1.07 \times 10^{-3}\right)$.

same in all three cases: the joint action of Coulomb correlations and elastic scattering, which leads to the result $S_{I}(0)$ $=S_{V}(0)$ [as shown in Fig. 9(a) in the 3D case], where $S_{V}(0) / 2 q I$ under perfect diffusive regime is a function of the dimensionality of momentum space.

In a recent work, Beenakker ${ }^{52}$ has developed an analytical theory able to explain the dependence of $\gamma$ on $d$ in nondegenerate diffusive conductors and has provided a close expression for $\gamma$ as a function of $d$. In particular, the theory predicts for $\gamma$ the values of 0.34 and 0.51 for, respectively, the 3D and 2D cases, in agreement with present findings. From that analysis, Beenakker concluded that the proximity of $\gamma$ to $1 / d$ is accidental. Indeed, in the 1D case we obtain a value of 0.7 , far from the full shot-noise value. In this $1 \mathrm{D}$ case his estimations for $\gamma$ provide a value of 0.92 , the discrepancy with respect to our result presumably due to the omission of the diffusion term in his calculations, which is especially important for low values of $d .^{63}$

For reason of completeness, we have finally analyzed the possible influence of an anisotropic elastic scattering on transport and noise in the 1D case. Figure 12 reports $\gamma$ and the current as a function of $\ell / L$ for three different values of $P_{b}: 0.5$ (isotropic case), 0.25 and 0.10 (less pronounced back-scattering). The noise results differ only in the quasiballistic regime, where the current remains nearly constant. Once the diffusive regime is achieved, the current decreases linearly with $\ell / L$ and $\gamma$ takes the same value of about 0.7 independently of the degree of scattering anisotropy. As ex- 


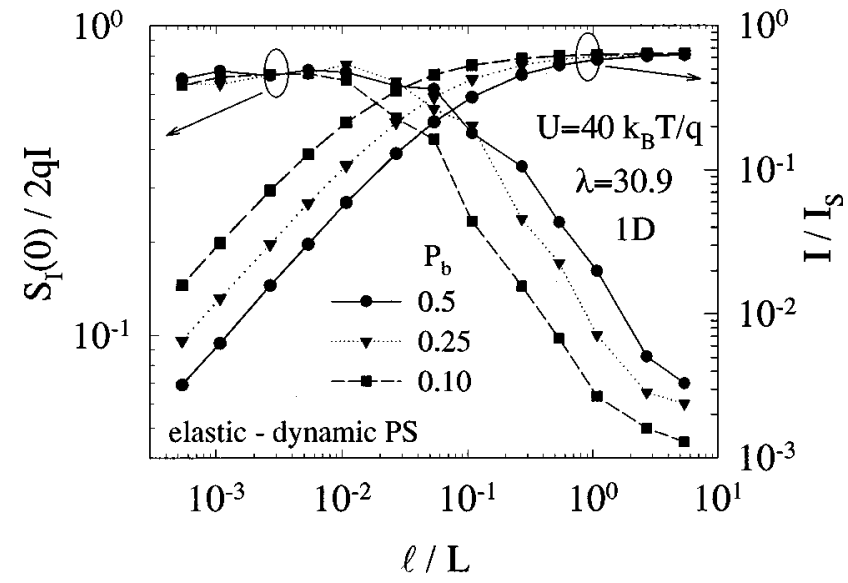

FIG. 12. Shot-noise suppression factor and normalized current vs the ballistic parameter $\ell / L$ calculated within the dynamic PS scheme for elastic scattering in the case of a 1D momentum space. Different curves refer to the reported values of the probability of scattering in the backward direction $P_{b}$. The applied voltage is $U$ $=40 k_{B} T / q$.

pected, we have found that the smaller the value of $P_{b}$ the wider the quasiballistic range, and for a given value of $\ell / L$ in this range the stronger the suppression, as corresponds to closer ballistic conditions.

\section{CONCLUSIONS}

We have provided a microscopic analysis of shot-noise suppression in nondegenerate diffusive conductors. To this purpose, the carrier dynamics in the active region of a semiconductor structure under the influence of elastic or inelastic scattering has been simulated by using an ensemble MC technique self-consistently coupled with a PS. The essential role played by long-range Coulomb interaction on the shotnoise suppression factor $\gamma$ has been demonstrated, since no suppression is found in the absence of the self-consistent potential fluctuations.

We have analyzed shot-noise suppression in the region of crossover from ballistic to diffusive transport regimes. In the diffusive regime a value of $\gamma$ independent of sample length is not achieved until a significant energy redistribution among momentum directions takes place. For high voltages $q U / k_{B} T \gg 1$ and long samples $\ell / L \ll 1$, in the elastic case shot noise is found to be suppressed to a $1 / 3$ value, while in the inelastic case we have found a stronger suppression the higher the applied voltage. Noticeably, in the perfect diffusive regime $\gamma$ is found to be independent of carrier injecting statistics, which implies that in this regime the noise is just a property of the sample.

Our results show that neither phase coherence nor Fermi statistics are necessary for the appearance of the 1/3 suppression factor in an elastic diffusive conductor. In our model, the appearance of this factor requires the simultaneous fulfillment of the three following conditions: $\ell / L \ll 1, \lambda \gg 1$, and $q U / k_{B} T \gg 1$. The first implies perfect diffusive regime, the second strong space-charge effects, and the third very farfrom-equilibrium conditions. Inelastic scattering is found to further contribute in suppressing shot noise, by reducing it to values close to thermal Nyquist noise under strong dissipative conditions. However, for this suppression to take place it is necessary to have the presence of long-range Coulomb interaction.

The action of Coulomb repulsion in suppressing shot noise takes place through the reduction of the contributions associated with carrier-number fluctuations to the total noise spectral density. In particular, the compensation between number and velocity-number terms implies that the total noise is finally determined just by the contribution of velocity fluctuations.

In the elastic case, $\gamma$ depends on the momentum space dimensionality, the suppression being less pronounced the lower the dimension of momentum space. This fact spoils the possible universality of the $1 / 3$ reduction found in the $3 \mathrm{D}$ nondegenerate case. ${ }^{5}$ Moreover, for a given dimensionality the consideration of an energy-dependent scattering rate can also lead to different suppression factors. ${ }^{52,63,64}$

We believe that the present investigation can provide a stimulus for an experimental verification of some of the reported results. As a valuable improvement we aim at including degenerate statistics in a further step.

\section{ACKNOWLEDGMENTS}

We thank R. Landauer for providing us a copy of his manuscript prior to publication. We gratefully acknowledge the support from the Dirección General de Enseñanza Superior e Investigación through the project PB97-1331, and the Physics of Nanostructures project of the Italian Ministero dell' Universitá e della Ricerca Scientifica e Tecnologica (MURST).
${ }^{1}$ A. van der Ziel, Noise, Source, Characterization, Measurement (Prentice-Hall, Englewood Cliffs, NJ, 1970).

${ }^{2}$ A. van der Ziel, Noise in Solid State Devices and Circuits (Wiley, New York, 1986).

${ }^{3}$ Sh. Kogan, Electronic Noise and Fluctuations in Solids (Cambridge University Press, Cambridge, England, 1996).

${ }^{4}$ M. J. M. de Jong and C. W. J. Beenakker, in Mesoscopic Electron Transport, Vol. 345 of NATO Advanced Study Institute, Series E: Applied Science, edited by L. P. Kowenhoven, G. Schön, and L. L. Sohn (Kluwer, Dordrecht, 1997), p. 225.

${ }^{5}$ R. Landauer, Nature (London) 392, 658 (1998).

${ }^{6}$ W. Schottky, Ann. Phys. (Leipzig) 57, 541 (1918).
${ }^{7}$ I. O. Kulik and A. N. Omel'yanchuk, Fiz. Nizk. Temp. 10, 305 (1984) [Sov. J. Low Temp. Phys. 10, 158 (1984)].

${ }^{8}$ G. B. Lesovik, Pis'ma Zh. Éksp. Teor. Fiz. 49, 513 (1989) [JETP Lett. 49, 592 (1989)].

${ }^{9}$ L. Chen and C. Ting, Phys. Rev. B 43, 4534 (1991).

${ }^{10}$ J. H. Davies, P. Hyldgaard, S. Hershfield, and J. W. Wilkins, Phys. Rev. B 46, 9620 (1992).

${ }^{11}$ M. J. M. de Jong and C. W. J. Beenakker, Phys. Rev. B 51, 16867 (1995).

${ }^{12}$ G. Iannaccone, M. Macucci, and B. Pellegrini, Phys. Rev. B 55, 4539 (1997). 
${ }^{13}$ C. W. J. Beenakker and M. Büttiker, Phys. Rev. B 46, 1889 (1992).

${ }^{14}$ K. E. Nagaev, Phys. Lett. A 169, 103 (1992).

${ }^{15}$ K. E. Nagaev, Phys. Rev. B 52, 4740 (1995).

${ }^{16}$ V. I. Kozub and A. M. Rudin, Phys. Rev. B 52, 7853 (1995).

${ }^{17}$ A. Shimizu and M. Ueda, Phys. Rev. Lett. 69, 1403 (1992).

${ }^{18}$ R. C. Liu and Y. Yamamoto, Phys. Rev. B 50, 17411 (1994).

${ }^{19}$ Y. P. Li, A. Zaslavsky, D. C. Tsui, M. Santos, and M. Shayegan, Phys. Rev. B 41, 8388 (1990).

${ }^{20}$ Y. P. Li, D. C. Tsui, J. J. Heremans, J. A. Simmons, and G. W. Weimann, Appl. Phys. Lett. 57, 774 (1990).

${ }^{21}$ F. Liefrink, J. I. Dijkhuis, M. J. M. de Jong, L. W. Molenkamp, and H. van Houten, Phys. Rev. B 49, 14066 (1994).

${ }^{22}$ H. C. Liu, J. Li, G. C. Aers, C. R. Leavens, M. Buchanan, and Z. R. Wasilewski, Phys. Rev. B 51, 5116 (1995).

${ }^{23}$ H. Birk, M. J. M. de Jong, and C. Schönenberger, Phys. Rev. Lett. 75, 1610 (1995).

${ }^{24}$ M. Reznikov, M. Heiblum, H. Shtrikman, and D. Mahalu, Phys. Rev. Lett. 75, 3340 (1995).

${ }^{25}$ A. Kumar, L. Saminadayar, D. C. Glattli, Y. Jin, and B. Etienne, Phys. Rev. Lett. 76, 2778 (1996)

${ }^{26}$ A. H. Steinbach, J. M. Martinis, and M. H. Devoret, Phys. Rev. Lett. 76, 3806 (1996).

${ }^{27}$ M. Henny, H. Birk, R. Huber, C. Strunk, A. Bachtold, M. Küger, and C. Schönenberger, Appl. Phys. Lett. 71, 773 (1997).

${ }^{28}$ R. J. Schoelkopf, P. J. Burke, A. A. Kozhevnikov, D. E. Prober, and M. J. Rooks, Phys. Rev. Lett. 78, 3370 (1997).

${ }^{29}$ H. Pothier, S. Guéron, N. O. Birge, D. Esteve, and M. H. Devoret, Phys. Rev. Lett. 79, 3490 (1997).

${ }^{30}$ M. Henny, S. Oberholzer, C. Strunk, and C. Schönenberger, Phys. Rev. B 59, 2871 (1999).

${ }^{31}$ B. J. Thompson, D. O. North, and W. A. Harris, RCA Rev. 4, 269 (1940); 4, 441 (1940).

${ }^{32} \mathrm{M}$. Büttiker, in 14th International Conference on Noise in Physical Systems and 1/f Fluctuations, edited by V. Bareikis and R. Katilius (World Scientific, Singapore, 1995), p. 35.

${ }^{33}$ R. Landauer and Th. Martin, Physica B 175, 167 (1991).

${ }^{34}$ Th. Martin and R. Landauer, Phys. Rev. B 45, 1742 (1992).

${ }^{35}$ R. Landauer, Phys. Rev. B 47, 16427 (1993).

${ }^{36}$ R. Landauer, Physica B 227, 156 (1996); Microelectron. Eng. (to be published).

${ }^{37}$ F. Green and M. P. Das, cond-mat/9809339 (unpublished).

${ }^{38}$ Y. Naveh, D. V. Averin, and K. K. Likharev, Phys. Rev. Lett. 79, 3482 (1997).

${ }^{39}$ K. E. Nagaev, Phys. Rev. B 57, 4628 (1998).

${ }^{40}$ Y. Naveh, D. V. Averin, and K. K. Likharev, Phys. Rev. B 59, 2848 (1999).

${ }^{41}$ Y. Naveh, D. V. Averin, and K. K. Likharev, Phys. Rev. B 58, 15371 (1998).

${ }^{42}$ K. E. Nagaev, Phys. Rev. B 58, R7512 (1998).

${ }^{43}$ T. González, O. M. Bulashenko, J. Mateos, D. Pardo, and L. Reggiani, Phys. Rev. B 56, 6424 (1997).

${ }^{44}$ O. M. Bulashenko, J. Mateos, D. Pardo, T. González, L. Reggiani, and J. M. Rubí, Phys. Rev. B 57, 1366 (1998).

${ }^{45}$ T. González, J. Mateos, D. Pardo, O. M. Bulashenko, and L. Reggiani, Semicond. Sci. Technol. 13, 714 (1998).

${ }^{46}$ R. C. Liu, P. Eastman, and Y. Yamamoto, Solid State Commun. 102, 785 (1997).
${ }^{47}$ T. González, C. González, J. Mateos, D. Pardo, L. Reggiani, O. M. Bulashenko, and J. M. Rubí, Phys. Rev. Lett. 80, 2901 (1998).

${ }^{48}$ Yu. V. Nazarov, Phys. Rev. Lett. 73, 134 (1994).

${ }^{49}$ Ya M. Blanter and M. Büttiker, Phys. Rev. B 56, 2127 (1997).

${ }^{50}$ E. V. Sukhorukov and D. Loss, Phys. Rev. Lett. 80, 4959 (1998).

${ }^{51}$ E. V. Sukhorukov and D. Loss, Phys. Rev. B 59, 13054 (1999).

${ }^{52}$ C. W. J. Beenakker, Phys. Rev. Lett. 82, 2761 (1999).

${ }^{53}$ T. González and D. Pardo, J. Appl. Phys. 73, 7453 (1993).

${ }^{54}$ The space charge inside the active region would be balanced by thin positive charge layers inside the contacts at the boundary with the sample (just surface charge in the case of very high $n_{c}$, similar to a metal contact), which are not considered in the simulation. Therefore, the effect of possible charge fluctuations at the contacts is not included in the calculation of the current. In any case, this effect is expected to appear at high frequencies (comparable with those of the plasma) while we are mostly interested in the low-frequency region of the noise spectrum.

${ }^{55}$ For a discussion on such a similarity, see M. A. Lampert and P. Mark, Current Injection in Solids (Academic, New York, 1970).

${ }^{56}$ In particular, a significant inhomogeneity in the charge distribution is present, as shown in Ref. 45 in the ballistic case.

${ }^{57}$ At decreasing $\tau$ the accuracy of the values of $\left\langle\Delta N^{2}\right\rangle /\langle N\rangle$ in the static scheme is lower. This explains the dispersion of the plotted results for $\ell / L<10^{-2}$. To improve the accuracy of the results in this range, it would be necessary to consider much longer (not presently affordable) simulation times.

${ }^{58}$ In a previous work [T. González, O. M. Bulashenko, J. Mateos, D. Pardo, L. Reggiani, and J. M. Rubí, Semicond. Sci. Technol. 12, 1053 (1997)], due to the oversight of a poorly pronounced long-time tail in the autocorrelation function associated with carrier-number fluctuations, we erroneously affirmed that in the diffusive regime the results for $S_{I}(0)$ obtained within the static PS scheme coincided with those of the dynamic PS scheme and did not follow $2 q I$.

${ }^{59}$ At decreasing voltages the determination of $S_{I}(0)$ can be carried out without major difficulties, however the lowest value reported for the current corresponds to $I / I_{S}=10^{-4}$. Indeed, below this value the statistical resolution of the simulation is no longer sufficient and the values of the current become not reliable. For $q U<k_{B} T$ we expect that the $I-U$ characteristic of the structures becomes linear because of the presence of two injecting contacts.

${ }^{60}$ Equation (2) describes correctly the thermal noise of the present structure, since the nonlinearity of the $I-U$ characteristic is taken into account through the factor $\langle N\rangle /\langle N\rangle_{0}$, which depends on the applied voltage.

${ }^{61}$ L. Reggiani, T. Kuhn, and L. Varani, Appl. Phys. A: Solids Surf. 54, 411 (1992).

${ }^{62}$ The appearance of the 1/3 factor in the 1D MC simulation of Ref. 46 does not contradict present results because there it was due to the effect of Pauli exclusion principle, while here the correlation between electrons comes from their Coulomb repulsion.

${ }^{63}$ H. Schomerus, E. G. Mishchenko, and C. W. J. Beenakker, Phys. Rev. B (to be published 15 August 1999).

${ }^{64}$ K. E. Nagaev cond-mat/9812357 (unpublished). 